\section{Drs. Littlejohn and Guymer reply}

\section{To the Editor:}

We thank Dr. Kapur for the comments ${ }^{1}$ on our recent editorial ${ }^{2}$. Many patients who have minor injuries in motor vehicle accidents can progress to develop significant and persistent pain. Often these patients fulfill criteria for fibromyalgia (FM), which is the most common chronic pain phenotype $^{3}$. The presence of psychological distress occurring in this context is the key to subsequent development of chronic musculoskeletal pain $^{4,5}$.

In these situations, it is important to "get the diagnosis right" because FM is associated with central pain while whiplash implies ongoing peripheral nociceptive pain. The term "whiplash" is an emotive term because it implies that the neck has been forcefully traumatized by the motor vehicle accident, thus implying ongoing injury. The term "fibromyalgia" is descriptive and defined by robust clinical criteria ${ }^{3,6}$. The central pain of FM has its origins in changes in control pathways linking the brain to the spinal $\operatorname{cord}^{7}$. There is subsequent increased sensitivity that upregulates incoming sensory inputs from a variety of sources including otherwise innocuous mechanoreceptor input that "gains access" to pain pathways $^{8}$. Other sensory inputs are also amplified, including light, noise, tinnitus, and bowel and bladder sensations. We suggest that emotional and distress factors, acting on background genetic and psychological vulnerability factors, associate with these changes in central pain control.

In regard to central sensitization, we feel that this in FM is occurring as a "top-down" phenomenon attributable to primary changes in the brain and spinal cord rather than being peripherally stimulated, as would occur in experimental models ${ }^{8}$. Further, the "top-down" mechanisms associated with central sensitization in FM may be persistent and cause ongoing symptoms over decades, although significant fluctuation and remission can occur in many people, congruent with the known plasticity of the central nervous system's function.

Background psychosocial stressors are common in FM, but many patients have onset of the clinical phenotype of FM following a specific trigger ${ }^{4}$. Such triggers are more often than not of a psychological type, but can include viral infection and physical trauma ${ }^{5,7}$. In the latter situation, psychological factors often accompanying the physical trauma can be key triggers rather than the physical trauma itself.

In this setting, litigation usually adds to the emotional distress of the individual. This and other societal factors may inadvertently promote the FM mechanism. Whether such patients are labeled as having posttraumatic stress disorder (PTSD) will depend on the specific criteria that are used. The symptoms of FM exist on a spectrum ranging from low through severe, which is similar to the spectrum of emotional distress. Thresholds for diagnosis of either FM or PTSD are therefore somewhat arbitrary in these settings.

We feel that the majority of patients in this situation have abnormal neurophysiology, which causes the symptoms. There is a smaller percentage that will exaggerate their symptoms for the sake of monetary or other rewards. Consideration of this smaller subgroup of individuals should not negate the observations in the larger group.

In regard to military medicine, we agree that there is a long history of description of pain phenotypes under different names over a long period of time $e^{9,10}$. The majority of such individuals have chronic pain, fatigue, poor sleep, and cognitive dysfunction, as well as background emotional distress of various types. These descriptors equate to the current descriptions of FM.
We agree that in civilian life there is an increased propensity to develop central sensitization and subsequent chronic pain disorders in certain people and in certain circumstances. This is particularly so when there is interaction between medical and legal issues, such as may occur in motor vehicle accidents. We reiterate that accurate diagnosis of the clinical phenotype of FM in this context is important; abandonment of the term whiplash and whiplash-associated disorder is important and appropriate management of the pain condition as a central pain condition rather than a peripheral pain condition will produce the best outcomes for each individual.

All clinicians who see people with these problems are trying to find the best way forward for their patients. The clinical features in these patients are identifiable as a discrete syndrome. We do not feel that the label "fibromyalgia" belongs to a lobby group, but rather describes a well-defined condition that we need to appropriately identify, understand, and manage. There is a large body of knowledge and a high level of understanding of FM that needs to be applied to all patients, including those where the condition occurs in the nexus of medicine and the law.

GEOFFREY OWEN LITTLEJOHN, MPH, MD, FRACP, Professor of Medicine, Emeritus Director, Rheumatology, Monash University and Monash Health; EMMA KATHRYN GUYMER, MBBS, FRACP, Head of Fibromyalgia Clinic, Monash Health, Clayton, Australia.

Address correspondence to Dr. Littlejohn, Suite H, Monash Medical Centre, 246 Clayton Road, Clayton, Victoria, Australia.

E-mail: Geoff.littlejohn@monash.edu

\section{REFERENCES}

1. Kapur D. Whiplash and fibromyalgia. J Rheumatol 2014;41:2323-4

2. Littlejohn GO, Guymer EK. Whiplash: same elephant, different room. J Rheumatol 2014;41:411-3

3. Wolfe F, Clauw DJ, Fitzcharles MA, Goldenberg DL, Hauser W, Katz RS, et al. Fibromyalgia criteria and severity scales for clinical and epidemiological studies: a modification of the ACR Preliminary Diagnostic Criteria for Fibromyalgia. J Rheumatol 2011;38:1113-22.

4. Buskila D, Mader R. Trauma and work-related pain syndromes: risk factors, clinical picture, insurance and law interventions. Best Pract Res Clin Rheumatol 2011;25:199-207.

5. Jones GT, Nicholl BI, McBeth J, Davies KA, Morriss RK, Dickens $\mathrm{C}$, et al. Role of road traffic accidents and other traumatic events in the onset of chronic widespread pain: Results from a population-based prospective study. Arthritis Care Res 2011;63:696-701.

6. Littlejohn G. Fibromyalgia: honing fibromyalgia diagnosis. Nat Rev Rheumatol 2014;10:267-9.

7. Clauw DJ. Fibromyalgia: a clinical review. JAMA 2014; 311:1547-55.

8. Woolf CJ. Central sensitization: implications for the diagnosis and treatment of pain. Pain 2011;152:S2-15.

9. Clauw DJ. The "gulf war syndrome": implications for rheumatologists. J Clin Rheumatol 1998;4:173-4.

10. Wallace DJ. Fibromyalgia: unusual historical aspects and new pathogenic insights. Mt Sinai J Med 1984;51:124-31.

J Rheumatol 2014;41:11; doi:10.3899/jrheum.140753 